\title{
Accuracy-Guaranteed Bit-Width Optimization
}

\author{
Dong-U. Lee, Member, IEEE, Altaf Abdul Gaffar, Member, IEEE, Ray C. C. Cheung, Student Member, IEEE, \\ Oskar Mencer, Member, IEEE, Wayne Luk, Member, IEEE, and George A. Constantinides, Member, IEEE
}

\begin{abstract}
An automated static approach for optimizing bit widths of fixed-point feedforward designs with guaranteed accuracy, called MiniBit, is presented. Methods to minimize both the integer and fraction parts of fixed-point signals with the aim of minimizing the circuit area are described. For range analysis, the technique in this paper identifies the number of integer bits necessary to meet range requirements. For precision analysis, a semianalytical approach with analytical error models in conjunction with adaptive simulated annealing is employed to optimize the number of fraction bits. The analytical models make it possible to guarantee overflow/underflow protection and numerical accuracy for all inputs over the user-specified input intervals. Using a stream compiler for field-programmable gate arrays (FPGAs), the approach in this paper is demonstrated with polynomial approximation, RGB-to-YCbCr conversion, matrix multiplication, $B$-splines, and discrete cosine transform placed and routed on a Xilinx Virtex-4 FPGA. Improvements for a given design reduce the area and the latency by up to $26 \%$ and $12 \%$, respectively, over a design using optimum uniform fraction bit widths. Studies show that MiniBit-optimized designs are within $1 \%$ of the area produced from the integer linear programming approach.
\end{abstract}

Index Terms-Field-programmable gate arrays (FPGAs), finite word-length effects, fixed-point arithmetic, optimization methods, simulated annealing (SA).

\section{INTRODUCTION}

$\mathbf{O}$ NE OF the main objectives of hardware designers is to find the optimal design in terms of area, latency, throughput, and power consumption. Bit widths of signals are one of the parameters that designers can tweak to improve these metrics. In contrast to instruction processors, customizable hardware such as field-programmable gate arrays (FPGAs) and application-specific integrated circuits (ASICs) provide the freedom for bit widths optimized for a given application. However, hardware designers face increasing difficulties choosing the best bit widths. The objective is to find the minimal number of bits to represent a signal, while satisfying user-defined error constraints. A naive way to optimize bit widths is to manually evaluate various combinations and observe the output for each

Manuscript received July 19, 2005; revised October 6, 2005. This work was supported in part by the Jet Propulsion Laboratory, by Xilinx Inc., by the U.K. Engineering and Physical Sciences Research Council under Grants GR/N 66599, GR/R 55931, and GR/R 31409, and by the Croucher Foundation. This paper was recommended by Associate Editor R. Camposano.

D.-U. Lee is with the Electrical Engineering Department, University of California, Los Angeles, CA 90095 USA (e-mail: dongu@icsl.ucla.edu).

A. A. Gaffar and G. A. Constantinides are with the Department of Electrical and Electronic Engineering, Imperial College London, SW7 2BT U.K. (e-mail: altaf.gaffar@imperial.ac.uk; g.constantinides@imperial.ac.uk).

R. C. C. Cheung, O. Mencer, and W. Luk are with the Department of Computing, Imperial College London, SW7 2AZ U.K. (e-mail: r.cheung@ imperial.ac.uk; o.mencer@imperial.ac.uk;w.luk@imperial.ac.uk).

Digital Object Identifier 10.1109/TCAD.2006.873887 design. This technique, however, involves an enormous search space and is not practical for large designs.

Bit-width optimization is an NP-hard problem [1] and has been the focus of numerous research contributions, especially over the past few years. The work in this area can be classified in many different ways, and one such classification is static analysis versus dynamic analysis. Dynamic analysis [2]-[7] relies on the use of stimuli input signals. Though this approach provides bit widths closer to the optimal set for those particular stimuli when compared to static-analysis techniques, it can be problematic since a large set of stimuli signals is required to analyze a design with sufficient confidence, possibly leading to prohibitively long simulation times and without guarantees for alternative input stimuli encountered in practice. Static analysis [8]-[12] is believed to give more conservative bit-width estimates than dynamic analysis. Static analysis is often more attractive than dynamic analysis especially for large designs, since only the characteristics of the input signals are needed. In this paper, we adopt a static-analysis technique based on affine arithmetic (AA) [13] and analytical error models to optimize both ranges and precisions for the signals in a fixedpoint design.

Another way of classifying bit-width optimization involves an error metric. Most existing work is based on the signal-tonoise ratio (SNR) error criterion. The SNR criterion is popular with digital signal processing applications. On the other hand, many computer arithmetic and scientific applications require a maximum absolute error bound. This error metric is good for portability, especially when a module needs to be integrated into a larger design. Our criterion for evaluating the accuracy is the unit in the last place (ulp). The ulp of a fixed-point number with 8 bits of fraction bit width would be $2^{-8}$. Faithful rounding means that results are accurate to $1 \mathrm{ulp}$ (rounded to the nearest or the next nearest) and exact rounding means that results are accurate to $0.5 \mathrm{ulp}$ (rounded to the nearest). Exact rounding is difficult to achieve, due to a problem known as the Table maker's dilemma [14] and has a large area penalty [15], hence we opt for faithful rounding in this initial paper. Therefore, if the result has 8 fraction bits, our approach guarantees a maximum absolute error of less than or equal to $2^{-8}$.

The main contributions of this paper are:

1) analytical range and uniform fraction bit-width (UFB) determination, based on AA models introduced in [9];

2) multiple fraction bit-width (MFB) determination via adaptive simulated annealing (ASA), using error models and area cost functions with guaranteed maximum absolute error bounds;

3) demonstration of our approach with five case studies: polynomial approximation, RGB-to-YCbCr conversion, 


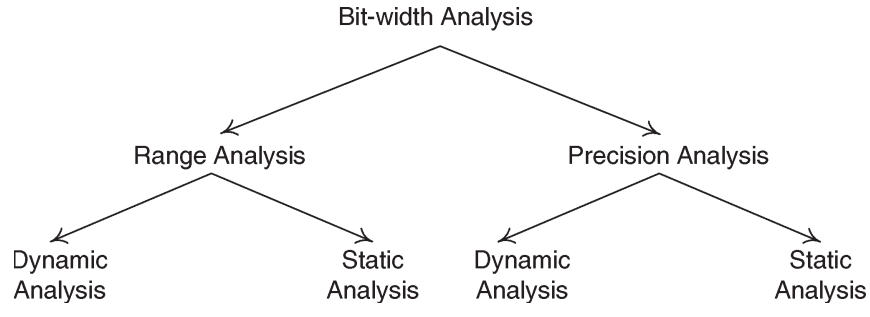

Fig. 1. Classification of bit-width analysis.

matrix multiplication, B-splines, and discrete cosine transform (DCT) realized in a Xilinx Virtex-4 FPGA;

4) only reported static bit-width-optimization technique that can guarantee 1-ulp maximum absolute error bound.

The rest of this paper is organized as follows. Section II discusses background material and related work. Section III presents an overview of our MiniBit bit-width-optimization approach. Section IV introduces AA. Sections V and VI present our range-analysis and precision-analysis steps. Section VII describes how our bit-width analysis can be extended to cover resource-sharing situations. Sections VIII and IX present our case studies and their results with MiniBit. Section $\mathrm{X}$ gives conclusions and future work.

\section{BACKGROUND}

As shown in Fig. 1, the problem of optimizing the design bit widths can be split into two parts: range analysis and precision analysis.

Range analysis involves studying the data range of the computation and ensuring that the signals in the design have enough bits to accommodate this range. Precision analysis involves analyzing the sensitivity of the output from a computation to slight changes in the bit widths, and more specifically, the sensitivity of an output to the computational precision within an arithmetic unit. For both range and precision analyses, we can apply a dynamic or a static-analysis method.

Dynamic-analysis methods evaluate the data flow graph (DFG) of the design using input stimuli signals. However, static-analysis methods propagate static characteristics of the inputs through the DFG, and hence no input stimuli are required. The input data dependence of dynamic analysis and the input data independence of static analysis have consequences on the results of the bit-width analysis.

As discussed next in the review of existing work in bit-width optimization, both these methods have certain advantages and disadvantages over each other.

\section{A. Existing Work}

A large body of work has been produced by Sung et al. [4], [5], which uses simulation-based techniques for range and precision bit-width optimizations. Their approach involves examining the mean and the standard deviations of the signals. Signals are grouped together during optimization to reduce simulation time. One of the drawbacks of this approach is that there is a danger of overflows for rare events.
The Fixed-point pRogrammIng DesiGn Environment (FRIDGE) [7] project provides a bit-width-optimization system for hardware/software codesign. FRIDGE uses intervalarithmetic-based range-propagation techniques for the range optimization and a simulation-based approach for the precision optimization. This approach relies on bit-width specification of some of the signals by the user and derives the remaining signal bit widths through what the authors describe as interpolation. In order to speed up the simulation time, the authors convert the hardware designs into integer-based American National Standards Institute (ANSI) C descriptions before simulation.

In [3], Cmar et al. use interval arithmetic (IA) for range bit-width determination and a dynamic method based on simulation for precision bit-width optimization. The simulation operates by simultaneously performing the same calculation in a reference floating point and a custom fixed-point format, and comparing the error between the two values. A heuristic is employed where the mean and the standard deviation of the error at each signal are examined for determining the precision bit widths.

In [6], Shi and Brodersen describe a statistical modeling method based on perturbation theory. For bit-width optimization, the method is designed to target optimization of algorithms used in communications applications. One feature of this approach is that it is data dependent due to its use of statistical modeling.

Gaffar et al. [2] use a mathematical technique known as automatic differentiation to perform precision bit-width optimization for both fixed-point and floating-point designs. Automatic differentiation is used to monitor the sensitivity of the output signals with respect to the intermediate signals. The proposed method requires less simulation time than conventional dynamic approaches.

Nayak et al. [16] describe a data-range-propagation method designed for the MATCH [17] system, which converts Matlab designs to FPGA design descriptions. Their range optimization relies on data range propagation, while precisions are optimized by forward propagation of errors through the DFG. A set of error transfer functions is proposed to determine the error contribution of each node.

Constantinides et al. propose Synoptix [8], an optimization technique targeting linear time-invariant digital signal processing systems using a novel resource binding technique. Synoptix uses a technique based on saturation arithmetic to perform the range bit-width optimization. In recent work [18], the proposed approach is extended to cover nonlinear systems, but this extension requires input stimuli to operate.

Fang et al. [9], [10] employ AA for modeling range and precision analyses. While the use of AA to model precision error is demonstrated, the authors do not use it to optimize the actual bit widths.

\section{B. Discussion}

In summary, for range analysis, most of the existing work considered use static analysis [3], [7]-[9], [16] with a few using dynamic analysis [2], [4]-[6]. For precision analysis, dynamic methods are employed in [2]-[7], while static methods are 


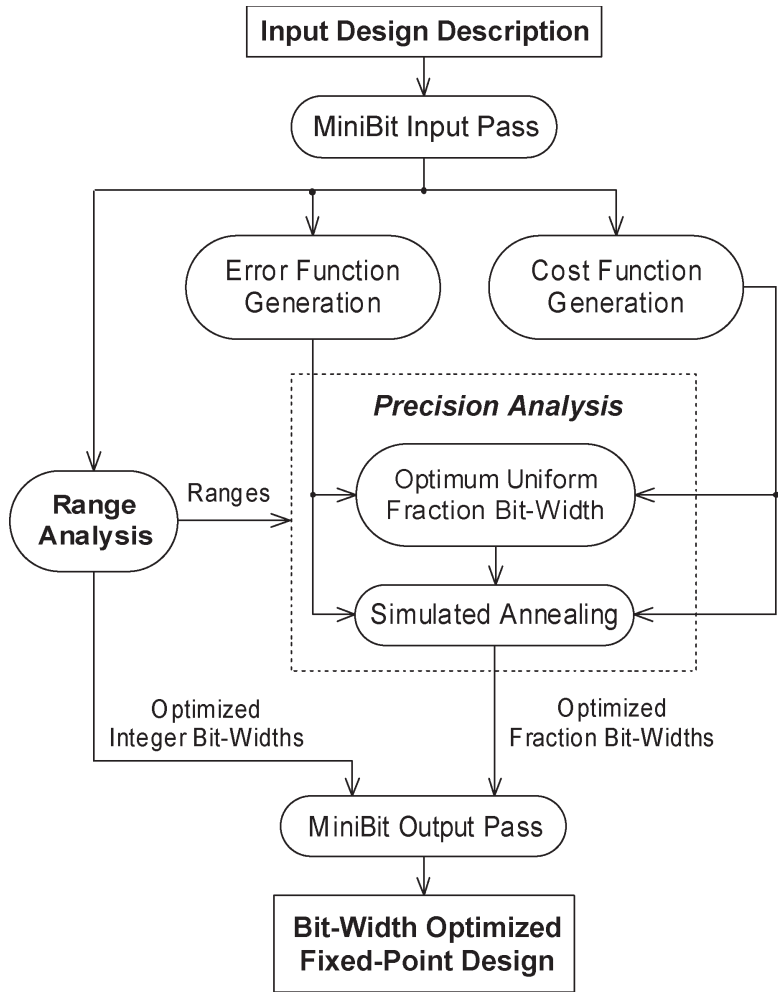

Fig. 2. Overview of MiniBit automated bit-width-optimization approach.

employed in [8], [9], and [16]. Static analysis, as we show in this paper, is capable of providing guaranteed analytical bounds on the output error.

Similar to the work by Fang et al., our proposed technique, MiniBit, uses static range analysis based on AA. However, we go a step further and tackle the problem of precision optimization as well. Our static precision-optimization approach is able to guarantee a maximum absolute error bound analytically, which is what differentiates our paper with the studies discussed in this section.

\section{OVERVIEW}

An overview of the MiniBit bit-width optimization framework is given in Fig. 2. MiniBit targets hardware designs using fixed-point representation. Fixed point is often preferred over floating point for hardware designs involving reasonable dynamic ranges due to its area and speed advantages. In fixedpoint representation, a real number is represented by two parts: an integer part, which represents the range, and a fraction part, which represents the precision. Two's complement representation is assumed. The range of a signal $x$ with $\mathrm{IB}_{x}$ integer bits and $\mathrm{FB}_{x}$ fraction bits is given by $\left[-\left(2^{\mathrm{IB}_{x}-1}-2^{-\mathrm{FB}_{x}}+1\right)\right.$, $\left.2^{\mathrm{IB}_{x}-1}-2^{-\mathrm{FB}_{x}}\right]$.

We divide the bit-width-optimization problem into two tasks: range analysis and precision analysis. Range analysis involves determining the integer bit widths (IBs) and precision analysis involves the determination of the required fraction bit widths (FBs) of fixed-point signals. Our approach is implemented as a series of compilation passes inside MiniBit, which is built on top of the BitSize bit-width analysis system [2]. The input to MiniBit is a design description in A Stream Compiler (ASC)
[19], C/C++, or Xilinx System Generator [20]. The MiniBit input pass uses this design description together with usersupplied information including the output error specification and the range of the input of values to perform range and precision analysis.

We first perform range analysis, then pass the range results to the precision-analysis phase. Precision analysis requires an error function and a cost function. The error function captures the output error as a function of the bit width of the signals of the design. The cost function returns the area cost as a function of the signal bit widths and their arithmetic operators.

Range analysis is performed via standard AA. Precision analysis operates in two phases: 1) Using the error function generated by MiniBit, we analytically find the optimum UFB, which means the FB for all signals are the same. The UFB serves as the initial set of parameters for the next phase. 2) We use both the error and cost functions to find the optimum MFBs, which, in contrast to UFB, means that the FBs of the signals can be different. MFBs aim at minimizing the area cost function while meeting the constraints of the error function. The MFBs are found by using ASA [21], [22].

Once the signals have been quantized, the ranges found in the range-analysis phase will slightly differ due to finite precision effects. Hence, range is a function of precision. However, as will be shown in Section VI, precision is a function of range. Since the actual range can marginally change after quantization, the range assumed during the precision-analysis phase can no longer be guaranteed to be perfectly accurate. In combination, these factors could, in theory, lead to increased IB requirements and/or increased FB requirements. Both of these potential problems can be addressed by using more conservative range estimates. However, these problems are highly unlikely to occur since: 1) only the ranges that are very close to a power of two can cause larger IB requirements and 2) due to the conservative nature of the precision analysis (which assumes maximum quantization errors can happen at all signals concurrently), the slight inaccuracy in the range will have a negligible impact. For the designs covered in this paper, we have not encountered such problems.

Having found the optimized IBs and FBs to each signal, the MiniBit output pass compares the outputs produced from our bit-width-optimized fixed-point design against the outputs produced from a software verification model. This step verifies that overflows/underflows do not occur and the user-specified error requirements are met. We finally synthesize and place and route the design to target technologies such as FPGAs or ASICs.

\section{AfFine Arithmetic}

IA [23] was invented in the 1960s by Moore to solve range problems, where each signal is represented by its interval. A signal $x$ is represented by the interval $\bar{x}=\left[x_{\min }, x_{\max }\right]$, meaning that the true value of $x$ lies between $x_{\min }$ and $x_{\max }$. Thus, for instance, the difference of two intervals $\bar{x}$ and $\bar{y}$ is expressed as

$$
\bar{x}-\bar{y}=\left[x_{\min }-y_{\max }, x_{\max }-y_{\min }\right] .
$$


The main disadvantage of IA is the assumption that all values of the arguments vary independently over the given intervals, potentially leading to drastic overestimation of the true range. As an extreme example, when evaluating the expression $x-x$, we get the interval $\bar{x}-\bar{x}=\left[x_{\min }-x_{\max }, x_{\max }-x_{\min }\right]$, which is twice as wide as the original interval $\bar{x}$, instead of $[0,0]$, which is the true range. This overestimation effect can accumulate along the computation chain, resulting in an "error explosion."

AA [13] is a recent refinement to IA to address this problem. AA captures all of the features of IA with one significant improvement: It keeps track of correlations among intervals. In AA, the uncertainty of a signal $x$ is represented by an affine form $\hat{x}$, which is a first-degree polynomial

$\hat{x}=x_{0}+x_{1} \varepsilon_{1}+x_{2} \varepsilon_{2}+\cdots+x_{n} \varepsilon_{n}, \quad$ where $\varepsilon_{i}=[-1,1]$.

Each $\varepsilon_{i}$ is an independent uncertainty source that contributes to the total uncertainty of the signal $x$. An ordinary IA interval $\bar{x}=\left[x_{\min }, x_{\max }\right]$ can be converted into an equivalent affine form $\hat{x}=x_{0}+x_{1} \varepsilon_{1}$ with

$$
x_{0}=\frac{x_{\max }+x_{\min }}{2} \quad x_{1}=\frac{x_{\max }-x_{\min }}{2} .
$$

The key feature of AA is that the sample noise symbol $\varepsilon_{i}$ can contribute to the uncertainty of other signals in the computation chain, keeping correlations between them. Returning to the previous example where IA overestimated, if $x$ has the affine form $\hat{x}=x_{0}+x_{1} \varepsilon_{1}$, then $\hat{x}-\hat{x}=0$, which is the correct result.

In AA form, we write: 1) addition/subtraction; 2) constant multiplication; and 3) addition/subtraction with a constant as

$$
\begin{gathered}
\hat{x} \pm \hat{y}=\left(x_{0} \pm y_{0}\right)+\sum_{i=1}^{n}\left(x_{i} \pm y_{i}\right) \varepsilon_{i} \\
c \hat{x}=\left(c x_{0}\right)+\sum_{i=1}^{n}\left(c x_{i}\right) \varepsilon_{i} \\
\hat{x} \pm c=\left(x_{0} \pm c\right)+\sum_{i=1}^{n} x_{i} \varepsilon_{i} .
\end{gathered}
$$

For multiplication, we get

$$
\begin{array}{r}
\hat{x} \hat{y}=\left(x_{0}+\sum_{i=1}^{n} x_{i} \varepsilon_{i}\right)\left(y_{0}+\sum_{i=1}^{n} y_{i} \varepsilon_{i}\right) \\
=x_{0} y_{0}+\sum_{i=1}^{n}\left(x_{0} y_{i}+y_{0} x_{i}\right) \varepsilon_{i}+Q \\
\text { where } Q=\left(\sum_{i=1}^{n} x_{i} \varepsilon_{i}\right)\left(\sum_{i=1}^{n} y_{i} \varepsilon_{i}\right) .
\end{array}
$$

The previous equation is not in affine form, due to the quadratic term $Q$. Hence, a conservative approximation is taken

$$
Q \approx u v \varepsilon_{n+1}, \quad \text { where } u=\sum_{i=1}^{n}\left|x_{i}\right| \quad v=\sum_{i=1}^{n}\left|y_{i}\right| .
$$

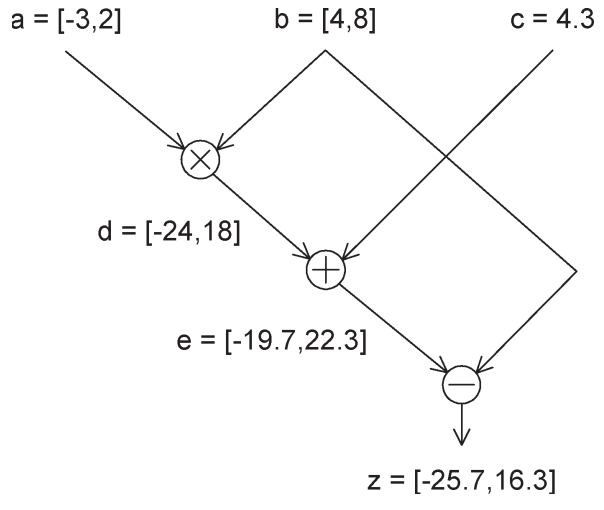

Fig. 3. Example circuit performing $z=a b+c-b$. Range of signal is shown in square brackets.

Affine forms for other elementary operations such as division and square root are given in [13]. It has been shown that AA gives tighter bounds than IA for both fixed-point [9] and floating-point designs [10].

\section{RANGe ANALYSIS}

The authors in [9] propose a single affine expression to capture both range and precision. However, we believe range and precision expressions should be kept separately. Precision is a function of range for operations such as multiplication and division; hence, the number of error terms $\varepsilon_{i}$ can easily explode. After range analysis, we obtain numerical values for the ranges; hence, the affine expressions for precisions remain manageable.

We use AA for the range analysis to minimize the IBs required for each signal. For instance, let us consider the evaluation of $z=a b+c-b$, as illustrated in Fig. 3. First, we assume the users know the exact range of all the input signals. Since we want to obtain the range for each signal, we set $d=a b$, $e=d+c$, and $y=e-b$. In affine form, we get

$$
\begin{array}{ll}
\hat{a}=-0.5+2.5 \varepsilon_{1} & \hat{b}=6+2 \varepsilon_{2} \\
\hat{c}=4.3 & \hat{d}=-3+15 \varepsilon_{1}-1 \varepsilon_{2}+5 \varepsilon_{3} \\
\hat{e}=1.3+15 \varepsilon_{1}-1 \varepsilon_{2}+5 \varepsilon_{3} & \hat{z}=-4.7+15 \varepsilon_{1}-7 \varepsilon_{2}+5 \varepsilon_{3} .
\end{array}
$$

Hence, the ranges of the signals are $d=[-24,18], e=$ $[-19.7,22.3]$, and $z=[-25.7,16.3]$. We perform range analysis on all signals for a given design and find the range for each signal. The IB required for a signal $x$ is computed with

$$
\begin{aligned}
\mathrm{IB}_{x}=\left\lceil\log _{2}\left(\max \left(\left|x_{\min }\right|,\left|x_{\max }\right|\right)\right)\right\rceil+\alpha \\
\text { where } \alpha= \begin{cases}1, & \bmod \left(\log _{2}\left(x_{\max }\right), 1\right) \neq 0 \\
2, & \bmod \left(\log _{2}\left(x_{\max }\right), 1\right)=0 .\end{cases}
\end{aligned}
$$

However, AA does not always lead to a better range estimation than IA. For instance, applying IA to the example, we obtain $d=[-24,16]$, which is narrower than the AA result. This is mainly due to the suboptimal fitting of an affine expression, which is not due to the affine approach itself. 


\section{PReCision ANALYSis}

We use AA for precision analysis in a similar fashion as for range analysis. There are two main ways to quantize a signal: truncation and round to nearest. Truncation and round to nearest can cause a maximum error of $2^{-\mathrm{FB}}(1 \mathrm{ulp})$ and $2^{-\mathrm{FB}-1}$ ( 0.5 ulp), respectively. Truncation chops bits off the least significant bits and requires no extra hardware resources. Round to nearest involves a small adder followed by truncation. For simplicity, we shall perform round to nearest throughout this paper. Hence, the quantized version $\tilde{x}$ of a signal $x$ is given in affine form by

$$
\tilde{x}=x+2^{-\mathrm{FB}_{\tilde{x}}-1} \varepsilon, \quad \text { where } \varepsilon=[-1,1]
$$

where $\mathrm{FB}_{\tilde{x}}$ is the $\mathrm{FB}$ of $\tilde{x}$. Hence, the error at $\tilde{x}$ due to finite precision effects is given by

$$
E_{\tilde{x}}=2^{-\mathrm{FB}_{\tilde{x}}-1} \varepsilon .
$$

For addition/subtraction, the affine error expression is given by

$$
\begin{aligned}
\tilde{z} & =\tilde{x} \pm \tilde{y}=x \pm y+E_{\tilde{x}} \pm E_{\tilde{y}}+2^{-\mathrm{FB}_{\tilde{z}}-1} \varepsilon_{3} \\
\Rightarrow E_{\tilde{z}} & =E_{\tilde{x}}+E_{\tilde{y}}+2^{-\mathrm{FB}_{\tilde{z}}-1} \varepsilon_{3} .
\end{aligned}
$$

For multiplication

$$
\begin{aligned}
\tilde{z} & =\tilde{x} \tilde{y}=x y+x E_{\tilde{y}}+y E_{\tilde{x}}+E_{\tilde{x}} E_{\tilde{y}}+2^{-\mathrm{FB}_{\tilde{z}}-1} \varepsilon_{3} \\
\Rightarrow E_{\tilde{z}} & =x E_{\tilde{y}}+y E_{\tilde{x}}+E_{\tilde{x}} E_{\tilde{y}}+2^{-\mathrm{FB}_{\tilde{z}}-1} \varepsilon_{3} .
\end{aligned}
$$

Assuming that the input signals need to be rounded, the application of the error models to the circuit in Fig. 3 is

$$
\begin{aligned}
E_{\tilde{a}} & =2^{-\mathrm{FB}_{\tilde{a}}-1} \varepsilon_{1} \\
E_{\tilde{b}} & =2^{-\mathrm{FB}_{\tilde{b}}-1} \varepsilon_{2} \\
E_{\tilde{c}} & =2^{-\mathrm{FB}_{\tilde{c}}-1} \varepsilon_{3} \\
E_{\tilde{d}} & =a E_{\tilde{b}}+b E_{\tilde{a}}+E_{\tilde{a}} E_{\tilde{b}}+2^{-\mathrm{FB}_{\tilde{d}}-1} \varepsilon_{4} \\
E_{\tilde{e}} & =E_{\tilde{d}}+E_{\tilde{c}}+2^{-\mathrm{FB}_{\tilde{e}}-1} \varepsilon_{6} \\
E_{\tilde{z}} & =E_{\tilde{e}}-E_{\tilde{b}}+2^{-\mathrm{FB}_{\tilde{z}}-1} \varepsilon_{7} .
\end{aligned}
$$

Note that $E_{\tilde{d}}$ would be at its maximum when the signals $a$ and $b$ are at their absolute maximum, i.e., $a=3$ and $b=8$.

Substituting the equations, we get the following maximum error at the output $\tilde{z}$ :

$$
\begin{aligned}
\max \left(E_{\tilde{z}}\right)= & 2^{-\mathrm{FB}_{\tilde{b}}}+2^{-\mathrm{FB}_{\tilde{a}}+2}+2^{-\mathrm{FB}_{\tilde{a}}-\mathrm{FB}_{\tilde{b}}-2} \\
& +2^{-\mathrm{FB}_{\tilde{d}}-1}+2^{-\mathrm{FB}_{\tilde{c}}-1}+2^{-\mathrm{FB}_{\tilde{e}}-1}+2^{-\mathrm{FB}_{\tilde{z}}-1} .
\end{aligned}
$$

For faithful rounding, the output error $E_{\tilde{z}}$ needs to be less than or equal to $1 \mathrm{ulp}$, i.e.,

$$
\begin{aligned}
2^{-\mathrm{FB}_{\tilde{z}}} \geq & \max \left(E_{\tilde{z}}\right) \\
\Rightarrow 2^{-\mathrm{FB}_{\tilde{z}}-1} \geq & 2^{-\mathrm{FB}_{\tilde{b}}}+2^{-\mathrm{FB}_{\tilde{a}}+2}+2^{-\mathrm{FB}_{\tilde{a}}-\mathrm{FB}_{\tilde{b}}-2} \\
& +2^{-\mathrm{FB}_{\tilde{d}}-1}+2^{-\mathrm{FB}_{\tilde{c}}-1}+2^{-\mathrm{FB}_{\tilde{e}}-1} .
\end{aligned}
$$

TABLE I

IBs, UFBs, AND MFBs to EXAMPLE Circuit IN FIg. 3

\begin{tabular}{c||c|c|c|c|c||c}
\hline \hline Signal & $\tilde{a}$ & $\tilde{b}$ & $\tilde{c}$ & $\tilde{d}$ & $\tilde{e}$ & $\tilde{z}$ \\
\hline \hline IB & 2 & 3 & 3 & 5 & 5 & 5 \\
\hline UFB & 12 & 12 & 12 & 12 & 12 & 8 \\
\hline MFB & 12 & 11 & 12 & 12 & 12 & 8 \\
\hline \hline
\end{tabular}

From (2), we see that the aim is to find the minimal FB for each signal, which satisfies the inequality and results in minimal circuit area. Since each FB is an integral value, the constraint space of this optimization problem is nonconvex. As a result, we choose the ASA package available in [22]. Traditional SA is very effective in discovering the global optimum, but its problem has been the slow convergence. ASA has been developed to statistically find the test global fit of a nonlinear constrained nonconvex cost function over a $D$-dimensional space. It permits adaptation to changing sensitivities in the multidimensional parameter space, thus allowing significantly faster convergence times.

In ASA, the user supplies a constraint function and a cost function. Error functions such as the inequality above are supplied as the constraint function. Since our aim is to minimize circuit area in this paper, we supply an area model of the circuit as a function of the signal bit widths as the cost function. In this area model, a full adder is assumed to be the unit area, i.e., the area for the addition $x+y$ is modeled with $\max \left(\mathrm{IB}_{x}+\right.$ $\left.\mathrm{FB}_{x}, \mathrm{IB}_{y}+\mathrm{FB}_{y}\right)$ and the area for the multiplication $x y$ is modeled with $\left(\mathrm{IB}_{x}+\mathrm{FB}_{x}\right)\left(\mathrm{IB}_{y}+\mathrm{FB}_{y}\right)$. These area models are derived to correspond with the operator area usage of our hardware compilation system (ASC) [19]. Other area models can be used to target different hardware compilers and device technologies.

The annealing process can be accelerated significantly by supplying good initial variable values (FBs in our case). Optimum uniform FBs are analytically computed and used as the initial variable values. For (2), substituting the FBs in the computation chain with a UFB

$$
2^{-\mathrm{FB}_{\tilde{z}-1}} \geq 2^{-\mathrm{UFB}}+2^{-\mathrm{UFB}+2}+2^{-2 \mathrm{UFB}-2}+3\left(2^{-\mathrm{UFB}-1}\right) .
$$

Let $\mathrm{FB}_{\tilde{z}}=8$ bits. Solving the equation for the minimum value of UFB that satisfies the inequality gives us UFB $=$ 12 bits, an analytical solution of the uniform bit-width selection problem.

The IBs, the UFBs, and the MFBs to the example circuit are summarized in Table I. The IBs are obtained by using the signal ranges found in Section V and (2), and the MFBs are computed by ASA. We observe that ASA is able to reduce the multiplication operand $b$ by 1 bit. The area cost of using UFBs and MFBs is found to be 244 and 230 units, respectively. By using MFBs, we have achieved an area saving of 6\%.

When using UFBs, we get a gap of $3.66 \times 10^{-4}$ between the actual error and the requested error, since the degree of freedom is one dimensional. However, with MFBs, we have a multidimensional degree of freedom, resulting in a smaller 


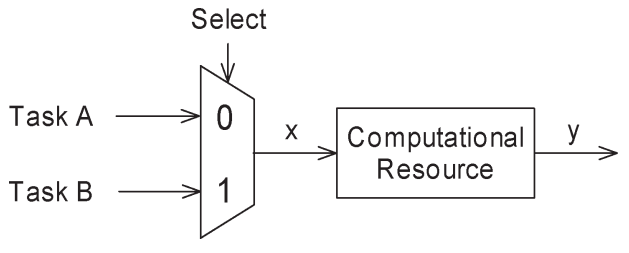

Fig. 4. Resource-sharing scenario: Tasks A and B time share computational resource.

error gap of $1.22 \times 10^{-4}$ and area cost. The differences between UFBs and MFBs are rather small for this example circuit, since the error gap is already rather small when using UFBs. We see more dramatic differences between UFBs and MFBs for designs with larger error gaps, as will be demonstrated with the case studies in Section IX.

\section{OPtIMIZATION Under Resource SHARING}

We often encounter a scenario such as the one depicted in Fig. 4 where we want to share a single resource for two (or more) tasks in a time-multiplexed manner. The range and precision analyses presented in the previous two sections can be trivially extended to cover these cases; signals that are shared among multiple tasks should be large enough to cover all tasks.

For the scenario in Fig. 4, we run range and precision analyses twice. In the first cycle, we assume that the select signal is set to zero, i.e., task $\mathrm{A}$ is in execution. In the second cycle, we assume that the select signal is set to one. After the two bitwidth analysis cycles, we obtain two sets of bit widths, each corresponding to a task data path. For all signals that are shared ( $x, y$, and all signals within the shared computational resource), we take the maximum of each signal's tuple of bit widths. For instance, if the signal $x$ has the following set: $\operatorname{IB}_{x}=\{2,4\}$ and $\mathrm{FB}_{x}=\{12,9\}$, we set $\mathrm{IB}_{x}=4$ and $\mathrm{FB}_{x}=12$. This scheme allows all signals to have sufficient bit widths for every task.

\section{CASe Studies}

For the five case studies, we assume that the inputs use the same FBs as the outputs. For the cases when multiple outputs are present, we use the same output precision for all outputs.

For these case studies, we develop a fully automated design flow that starts with designs captured in ASC [19] C++ syntax. All stages within MiniBit (Fig. 2) are written in C++ and integrated within the ASC system. The MiniBit input pass parses the input design description into an internal DFG representation for the range-analysis pass. The error-function generation pass produces an error model and the cost-function generation pass produces an area-cost model. The results from range analysis, together with the generated error function and cost function, are used to derive the UFB. Next, using ASA, we compute the MFBs. The MiniBit output pass converts the bit-width-optimized DFG representations back into ASC design description. The verification output-generation step produces a variable-bit-width-assigned design for simulation to verify the optimized results.
TABLE II

Number of AdDitions/SubTractions, Multiplications, AND SIgNALS TO BE OPTIMIZED

\begin{tabular}{c||c|c|c}
\hline \hline Case Study & Add/Sub & Mult & Signals \\
\hline \hline Degree 4 Poly & 4 & 4 & 12 \\
\hline RGB to YCbCr & 6 & 7 & 19 \\
\hline $2 \times 2$ Matrix Mult & 18 & 7 & 21 \\
\hline B-Splines & 15 & 6 & 22 \\
\hline $8 \times 8$ DCT & 32 & 32 & 55 \\
\hline \hline
\end{tabular}

\section{A. Polynomial Approximation}

We examine the degree-four polynomial for the approximation to $y=\log (1+x)$, where $x=[0,1)$. Horner's rule evaluates the polynomial

$$
y=\left(\left(c_{d} x+c_{d-1}\right) x+\cdots\right) x+c_{0}
$$

where $c_{0}, \ldots, c_{d}$ are the polynomial coefficients. The coefficients are obtained in a minimax sense to minimize the maximum absolute error.

\section{B. $\mathrm{RGB}$ to $\mathrm{YCbCr}$}

We consider the RGB-to-YCbCr color space converter specified by the JPEG 2000 standard [24]. The input signals R, G, and $\mathrm{B}$ are assumed to be eight-bit unsigned integers. Shifts are used for the multiplications by 0.5

$$
\left[\begin{array}{c}
\mathrm{Y} \\
\mathrm{Cb} \\
\mathrm{Cr}
\end{array}\right]=\left[\begin{array}{ccc}
0.299 & 0.587 & 0.114 \\
-0.16875 & -0.33126 & 0.5 \\
0.5 & -0.41869 & -0.08131
\end{array}\right]\left[\begin{array}{l}
\mathrm{R} \\
\mathrm{G} \\
\mathrm{B}
\end{array}\right]
$$

\section{Matrix Multiplication}

The $2 \times 2$ matrix multiplication using Strassen's algorithm [25] is considered, which is commonly used as a basic processing element for large matrix multiplications. We assume the elements of the input matrices are over $[0,1)$

$$
\left[\begin{array}{ll}
y_{00} & y_{01} \\
y_{10} & y_{11}
\end{array}\right]=\left[\begin{array}{ll}
a_{00} & a_{01} \\
a_{10} & a_{11}
\end{array}\right]\left[\begin{array}{ll}
b_{00} & b_{01} \\
b_{10} & b_{11}
\end{array}\right] .
$$

The four quadrants of the result matrix can be calculated as follows:

$$
\begin{array}{ll} 
& p_{0}=\left(a_{00}+a_{11}\right)\left(b_{00}+b_{11}\right) \\
y_{00}=p_{0}+p_{3}-p_{4}+p_{6} & p_{1}=\left(a_{10}+a_{11}\right) b_{00} \\
y_{01}=p_{2}+p_{4} & p_{2}=a_{00}\left(b_{01}-b_{11}\right) \\
y_{10}=p_{1}+p_{3} & p_{3}=a_{11}\left(b_{10}-b_{00}\right) \\
y_{11}=p_{0}+p_{2}-p_{1}+p_{5} & p_{4}=\left(a_{00}+a_{01}\right) b_{11} \\
& p_{5}=\left(a_{10}-a_{00}\right)\left(b_{00}+b_{01}\right) \\
& p_{6}=\left(a_{01}-a_{11}\right)\left(b_{10}+b_{11}\right) .
\end{array}
$$


TABLE III

Optimization Times AND ERRoR STATISTICS OF MFB DESigns (MFB RESUlTS) AND COMPARISONS BETWEEN UFB AND MFB DESIGNS (UFB/MFB COMPARISONS)

\begin{tabular}{|c|c|c|c|c|c|c|c|c|c|c|c|c|c|}
\hline \multicolumn{2}{|c|}{ Case Study } & \multicolumn{4}{|c|}{ MFB Results } & \multicolumn{8}{|c|}{ UFB/MFB Comparisons } \\
\hline \multirow{2}{*}{ Application } & \multirow{2}{*}{$\begin{array}{l}\text { Prec } \\
{[\mathrm{bits}]}\end{array}$} & \multirow{2}{*}{$\begin{array}{c}\text { Opt } \\
\text { Time }[\mathrm{s}]\end{array}$} & \multirow{2}{*}{$\begin{array}{c}\text { Max Err } \\
\text { [ulp] }\end{array}$} & \multirow{2}{*}{$\begin{array}{c}\text { Avg Err } \\
{[\text { ulp] }}\end{array}$} & \multirow{2}{*}{$\begin{array}{l}\text { SNR } \\
{[\mathrm{dB}]}\end{array}$} & \multicolumn{4}{|c|}{ Area [slices] } & \multicolumn{4}{|c|}{ Latency [ns] } \\
\hline & & & & & & UFB & MFB & Diff & Impr [\%] & UFB & MFB & Diff & $\operatorname{Impr}[\%]$ \\
\hline Degree 4 & 8 & 1.9 & 0.694 & 0.253 & 51.5 & 803 & 723 & 80 & 9.96 & 114.00 & 105.39 & 8.61 & 7.55 \\
\hline Polynomial & 16 & 2.0 & 0.731 & 0.256 & 99.5 & 1921 & 1797 & 124 & 6.45 & 168.55 & 151.81 & 16.74 & 9.93 \\
\hline \multirow{2}{*}{$\mathrm{RGB}$ to $\mathrm{YCbCr}$} & 8 & 8.9 & 0.662 & 0.260 & 97.2 & 1165 & 1132 & 33 & 2.83 & 37.47 & 36.95 & 0.52 & 1.39 \\
\hline & 16 & 9.7 & 0.793 & 0.272 & 144.9 & 1641 & 1602 & 39 & 2.38 & 50.26 & 48.83 & 1.43 & 2.85 \\
\hline $2 \times 2$ Matrix & 8 & 16.1 & 0.520 & 0.251 & 54.4 & 1896 & 1799 & 97 & 5.12 & 44.20 & 42.73 & 1.47 & 3.33 \\
\hline Multiplication & 16 & 19.5 & 0.528 & 0.247 & 102.5 & 4240 & 4072 & 168 & 3.96 & 59.22 & 56.14 & 3.08 & 5.20 \\
\hline \multirow{2}{*}{ B-Splines } & 8 & 27.7 & 0.716 & 0.267 & 49.8 & 1189 & 952 & 237 & 19.93 & 88.39 & 78.58 & 9.81 & 11.10 \\
\hline & 16 & 32.8 & 0.774 & 0.278 & 96.6 & 2652 & 2165 & 487 & 18.36 & 130.11 & 114.03 & 16.08 & 12.36 \\
\hline \multirow{2}{*}{$8 \times 8 \mathrm{DCT}$} & 8 & 154.3 & 0.702 & 0.254 & 103.1 & 5368 & 5217 & 151 & 2.81 & 54.83 & 50.73 & 4.10 & 7.48 \\
\hline & 16 & 179.1 & 0.708 & 0.257 & 151.3 & 7320 & 7167 & 153 & 2.09 & 66.39 & 59.42 & 6.97 & 10.50 \\
\hline
\end{tabular}

\section{B-Splines}

We examine uniform cubic B-splines, commonly used for image warping applications [26]. The B-spline basis functions $B_{0}, B_{1}, B_{2}$, and $B_{3}$ are defined by

$$
\begin{array}{ll}
B_{0}(u)=\frac{(1-u)^{3}}{6} & B_{2}(u)=\frac{-3 u^{3}+3 u^{2}+3 u+1}{6} \\
B_{1}(u)=\frac{3 u^{3}-6 u^{2}+4}{6} & B_{3}(u)=\frac{-u^{3}}{6}
\end{array}
$$

where $u=[0,1)$. For the implementation of this design, optimizations including shifts instead of multiplications, and sharing common intermediate results are carried out.

\section{E. Discrete Cosine Transform (DCT)}

We consider the $8 \times 8$ DCT implemented according to [27] A vector of input data $x_{0, \ldots, 7}$ can be transformed to DCT coefficients $y_{0, \ldots, 7}$ by

$$
\begin{aligned}
& {\left[\begin{array}{l}
y_{0} \\
y_{2} \\
y_{4} \\
y_{6}
\end{array}\right]=\left[\begin{array}{cccc}
c_{0} & c_{0} & c_{0} & c_{0} \\
c_{2} & c_{5} & -c_{5} & c_{2} \\
c_{0} & -c_{0} & -c_{0} & c_{0} \\
c_{5} & -c_{2} & c_{2} & -c_{5}
\end{array}\right]\left[\begin{array}{l}
x_{0}+x_{7} \\
x_{1}+x_{6} \\
x_{2}+x_{5} \\
x_{3}+x_{4}
\end{array}\right]} \\
& {\left[\begin{array}{l}
y_{1} \\
y_{3} \\
y_{5} \\
y_{7}
\end{array}\right]=\left[\begin{array}{cccc}
c_{1} & c_{3} & c_{4} & c_{6} \\
c_{3} & -c_{6} & -c_{1} & -c_{4} \\
c_{4} & -c_{1} & c_{6} & c_{0} \\
c_{6} & -c_{4} & c_{3} & -c_{1}
\end{array}\right]\left[\begin{array}{l}
x_{0}-x_{7} \\
x_{1}-x_{6} \\
x_{2}-x_{5} \\
x_{3}-x_{4}
\end{array}\right]}
\end{aligned}
$$

where $c_{0, \ldots, 7}$ are trigonometric constants. We use 8-bit unsigned integers for the elements in the input vector $x_{0, \ldots, 7}$.

\section{RESUlts}

We implement the five case studies with ASC for FPGAs [19]. The ASC code makes use of C++ syntax and ASC semantics, which allow the user to program on the architecture level, the arithmetic level, and the gate level. Designs are synthesized with ASC and placed and routed with Xilinx ISE 6.3 on a Xilinx Virtex-4 XC4VLX100-11 FPGA. The device

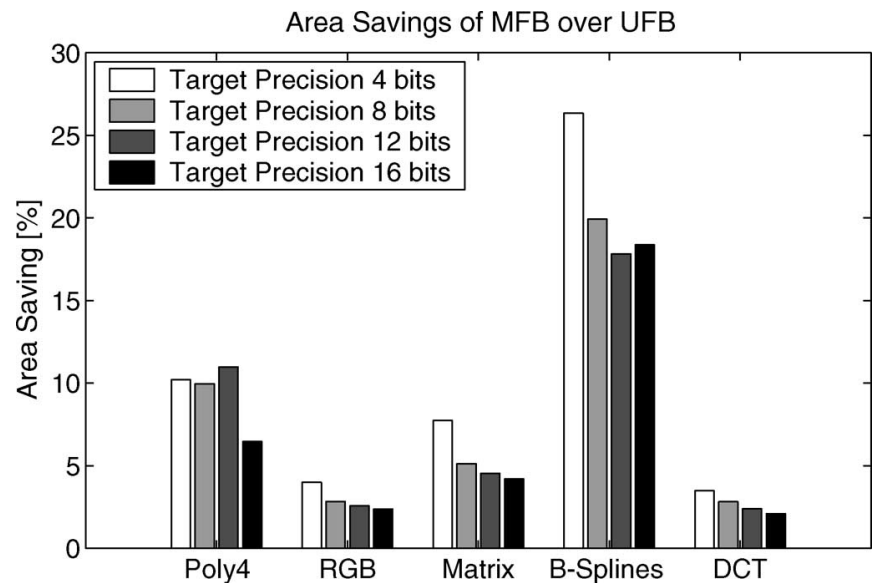

Fig. 5. Percentage area savings of MFB over UFB at different target precisions.

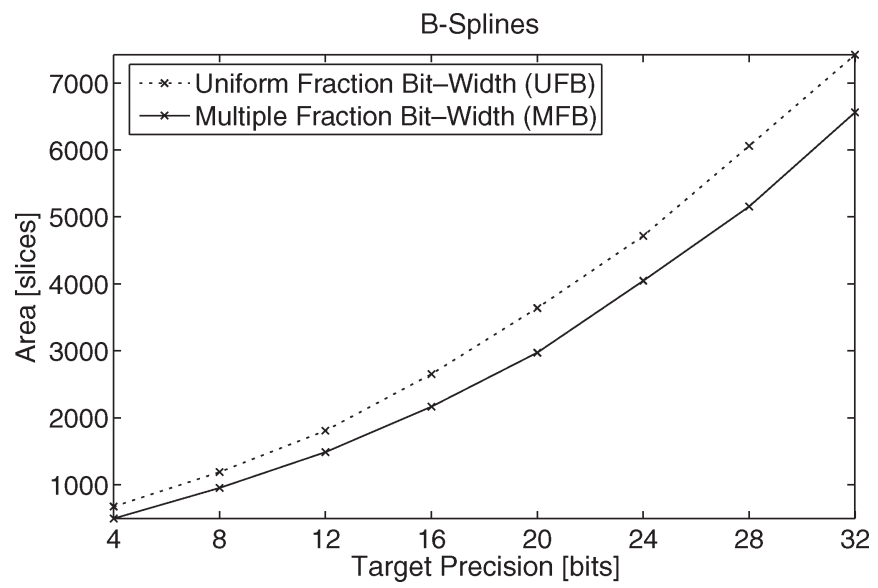

Fig. 6. Area variation for B-splines with increasing target precision.

contains user-programmable elements known as slices, dedicated multiply-and-add units, and embedded RAMs. In order to make fair comparisons, we implement designs using slices only and combinatorially without any pipelining. Table II shows the number of additions, subtractions, multiplications, and signals 
Target Precision 8 bits

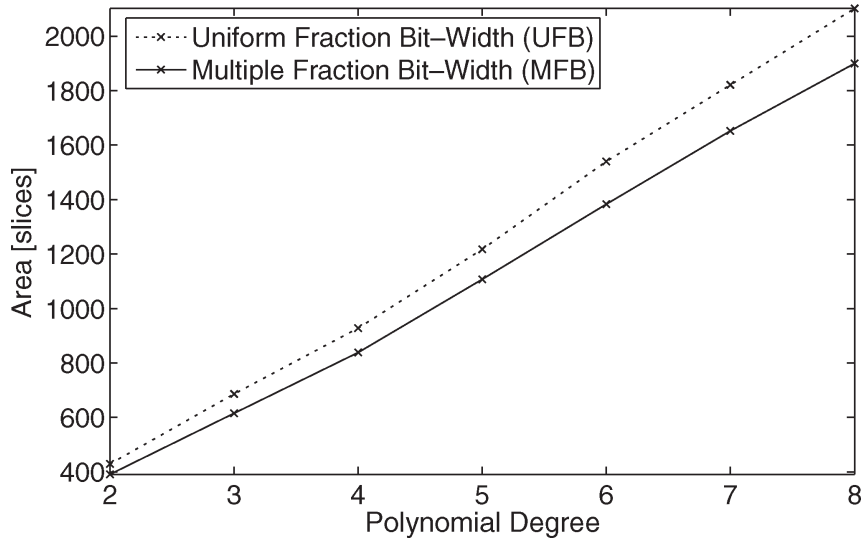

Fig. 7. Area variation for various polynomial degrees with target precision fixed at 8 bits.

to be optimized for the five case studies. We observe that the degree-four polynomial is the least complex and the $8 \times 8$ DCT has the most complexity.

\section{A. Comparisons of UFBs and MFBs}

Table III shows the optimization times and error statistics of MFB designs and comparisons between UFB and MFB designs. The UFB and MFB designs use the same number of integer bits for all signals, as computed in our range-analysis phase. The optimization times have been measured on an AMD Athlon XP 2600+ PC with 2-GB double data rate synchronous dynamic RAM (DDR-SDRAM) and include both range- and precision-analysis times. ASA is the dominant factor in the optimization process and we observe that more time is needed for designs with more signals, which is expected. Note that for all case studies, the optimization times are a matter of seconds.

The ulp errors and SNRs are computed by simulating MFBoptimized designs using random input vectors. The IEEE 64-bit double-precision floating point is assumed to be the true value for the error computations, since it is significantly more accurate than the precisions we are targeting. The maximum ulp errors for all designs are well below $1 \mathrm{ulp}$, indicating that the results are indeed faithfully rounded. Also, the average ulp error is less than 0.3 for all case studies. Looking at the area and speed comparisons, although we optimize designs for minimal area, the reduction in the multiplier and adder sizes leads to reductions in latencies as a by-product. We note that designs using MFBs are always smaller and faster than designs using UFBs. Some of the savings may seem rather small, but we get these savings for free, as the MFB designs have the same error bound as the UFB designs.

Fig. 5 shows the percentage area savings of MFB over UFB for the five case studies at different target precisions. Generally, we obtain increased relative savings for lower precisions. Another trend is that designs with deeper computation chains can benefit more with MFBs. The depths can be deduced by examining the latency (combinatorial delay) results in Table III. An area savings of up to $26 \%$ is achieved in the case of B-Splines, which has a deep computation chain.

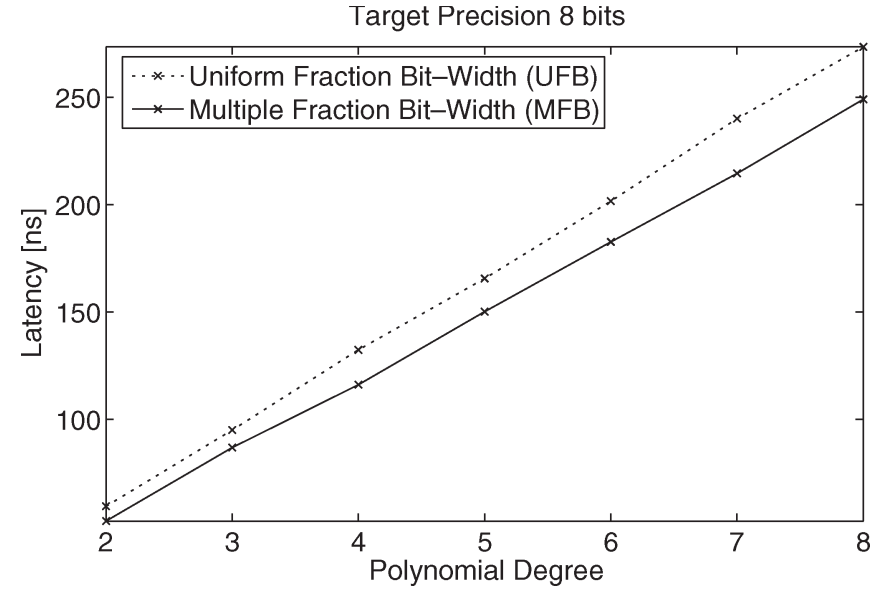

Fig. 8. Latency variation for various polynomial degrees with target precision fixed at 8 bits.

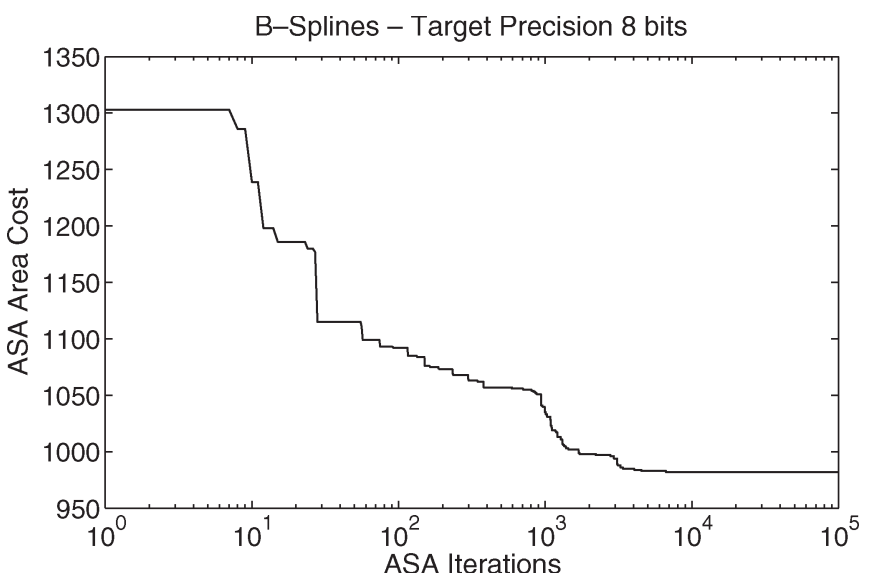

Fig. 9. ASA area-cost variation with ASA iterations for B-splines.

Fig. 6 shows the area variation for B-splines with increasing target precision. It can be seen that the area differences between UFB and MFB are increasing with the target precision. Figs. 7 and 8 show the area and latency variations for various polynomial degrees with target precision fixed at 8 bits. Since we use Horner's rule to evaluate polynomials, one extra degree causes one more adder and one more multiplier. In order to make a fair comparison, the coefficients are set to the number $\pi$ throughout. We can see that as we increase the depth of the computation chain (i.e., increase the polynomial degree), the area and latency differences between UFB and MFB increase.

Fig. 9 illustrates how the best ASA area cost varies with the number of iterations for B-splines with a target precision of 8 bits. ASA first starts with UFB, which results in a cost value of 1303 units. We observe that by increasing the number of iterations, we gradually approach a set of MFBs that give the lowest cost value, which is 982 units in this case. The annealing time to approach this optimum cost value is $23 \mathrm{~s}$.

\section{B. Comparisons of Different Range-Analysis Methods}

We examine the impact of using different range-analysis methods on designing the area and latency. Comparisons to the five case studies using simulations AA and IA for the range 
TABLE IV

ARea and Latency Comparisons Using Different Range-Analysis Methods for Target Precision of 8 Bits

\begin{tabular}{c||c|c|c|c|c|c}
\hline \hline \multirow{2}{*}{ Case Study } & \multicolumn{3}{c|}{ Area [slices] } & \multicolumn{3}{c}{ Latency [ns] } \\
\cline { 2 - 7 } & Simulation & Affine Arithmetic & Interval Arithmetic & Simulation & Affine Arithmetic & Interval Arithmetic \\
\hline \hline Degree 4 Poly & 701 & 723 & 732 & 103.21 & 105.39 & 107.37 \\
\hline RGB to YCbCr & 1109 & 1132 & 1143 & 35.17 & 36.95 & 38.82 \\
\hline $2 \times 2$ Matrix Mult & 1747 & 1799 & 1838 & 40.71 & 42.73 & 43.68 \\
\hline B-Splines & 937 & 952 & 978 & 76.01 & 78.58 & 89.86 \\
\hline $8 \times 8$ DCT & 5103 & 5217 & 5312 & 47.61 & 50.73 & 51.30 \\
\hline \hline
\end{tabular}

Target Precision 8 bits

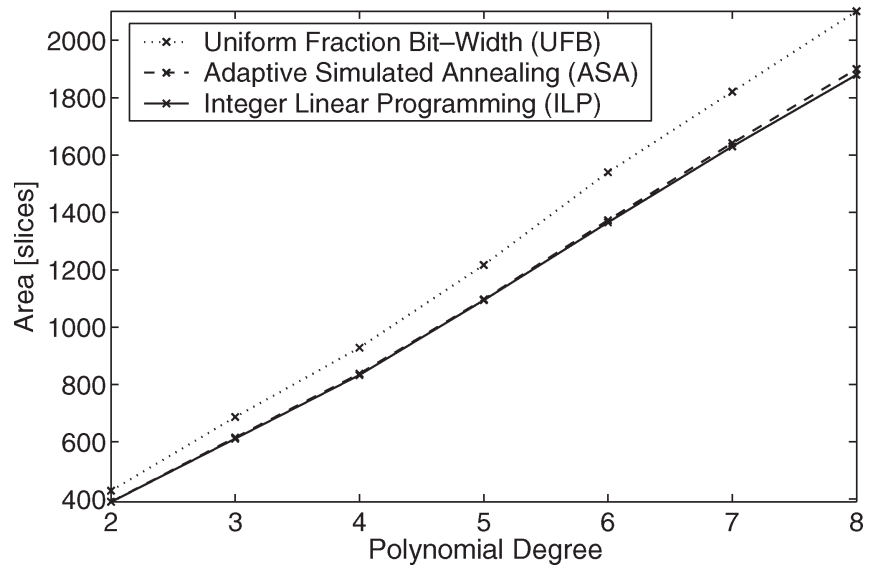

Fig. 10. Area comparison for various polynomial degrees with target precision fixed at 8 bits.

are shown in Table IV. The simulation-based range analysis is performed by feeding the designs with a large data set of random input samples and observing the data range. Although the simulation method gives smaller area and shorter latency, its drawback is that it can only guarantee overflow/underflow protection for the samples tested. It becomes quickly impractical for high input resolutions, since the run time increases exponentially with the resolution.

We also observe that AA gives slightly better results than IA. This is due to the fact that AA can exploit correlations between signals, as discussed in Section IV.

\section{Comparisons With Integer Linear Programming (ILP)}

In order to determine the optimality of the ASA solution used in MiniBit, we compare our results against the optimal bit widths allocated through ILP, which is known to give global optimum results. The ILP formulations are solved by using the ILOG CPLEX [28] solver. The optimization problem contains nonlinear functions that are converted into equivalent linear functions following the approach in [29]. The obtained ILP results are used as optimal solutions to judge the quality of the ASA results. The ILP solutions presented here take several hours to several days on an AMD Athlon XP 2600+ PC with 2-GB DDR-SDRAM.

First, we consider the comparison between ILP and ASA for different design complexities. Fig. 10 presents the results for optimizing degree-2 to degree-12 polynomials using ILP, ASA, and UFB solutions. Although the area savings of ILP over ASA

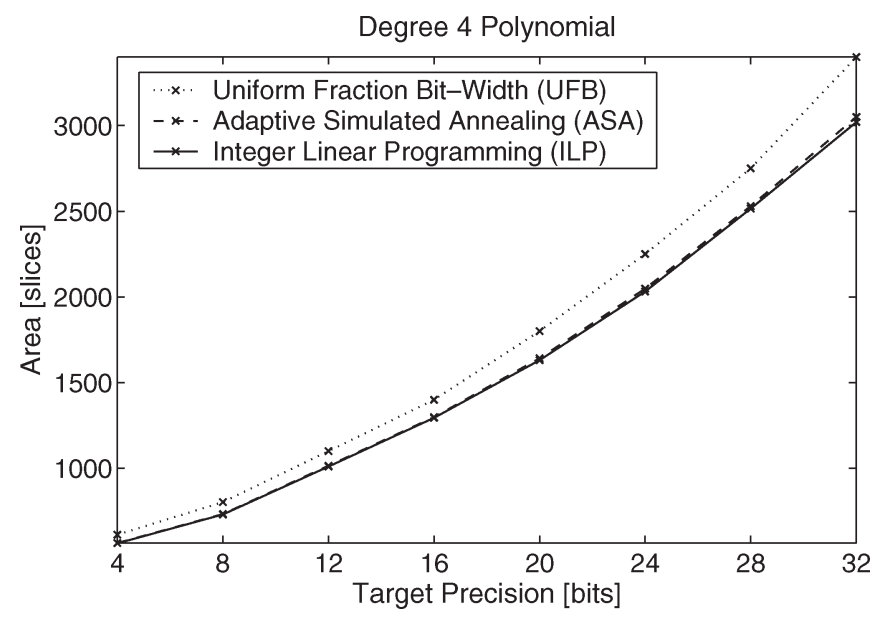

Fig. 11. Area variation for a degree-four polynomial with increasing target precision.

improves slightly with design complexity, they are less than $1 \%$. This indicates that with ASA, we can produce results that are close to the global optimum even with increasing design complexities.

Next, we study optimality of the ASA solution when the design constraint is changed using the degree-four polynomial design. Fig. 11 shows the area cost for optimizing the design with different target-precision requirements. We see a similar trend to Fig. 10, and again the differences are within $1 \%$.

We conclude that although ILP provides global optimal solutions, we can achieve near-optimal solutions using ASA with several orders of magnitude less run time.

\section{Conclusion}

We have presented MiniBit, an automated approach for optimizing bit widths of fixed-point designs with static analysis, for designing fixed-point hardware with guaranteed accuracy. We have described methods to minimize both the integer and the fraction parts of fixed-point signals based on AA. For precision analysis, we employ a semianalytical approach, where analytical error models in conjunction with ASA are used to find a local optimum number of fraction bits. The analytical models allow us to guarantee overflow/underflow protection and numerical accuracy for all possible inputs over the userspecified input intervals. We make the assumption that the maximum errors can happen at all nodes at the same time; however, in practice, this will perhaps never be the case. This assumption will often result in pessimistic bit widths. However, 
given that our approach can guarantee accuracy, we believe it is a small tradeoff to make.

Although our approach has been primarily designed for FPGA and ASIC applications, its principles could be applied to other applications such as fixed-point digital signal processors (DSPs). For DSPs, one could apply our range analysis to determine the IBs and use our precision analysis to calculate the maximum error bounds at the output of the computation chain. We can also apply MiniBit to configurable processors with extensible instructions for embedded applications such as Tensilica [30] by optimizing the bit widths of the custom instruction blocks.

Two limitations of our approach are: 1) the search space for ASA can be vast for large designs, leading to slow optimization times and 2) although this initial paper does not cover designs with a feedback loop, we can extend the error model by using the technique described in [9]. For future studies, we hope to use clustering techniques to optimize parts of a large design independently, which will result in suboptimal bit widths but faster optimization time. Moreover, we hope to extend MiniBit to cover floating-point arithmetic.

\section{ACKNOWLEDGMENT}

The authors would like to thank the anonymous reviewers for their remarks.

\section{REFERENCES}

[1] G. Constantinides and G. Woeginger, "The complexity of multiple wordlength assignment," Appl. Math. Lett., vol. 15, no. 2, pp. 137-140, 2001.

[2] A. Abdul Gaffar, O. Mencer, W. Luk, and P. Cheung, "Unifying bit-width optimisation for fixed-point and floating-point designs," in Proc. IEEE Symp. Field-Program. Custom Comput. Mach., 2004, pp. 79-88.

[3] R. Cmar, L. Rijnders, P. Schaumont, S. Vernalde, and I. Bolsens, "A methodology and design environment for DSP ASIC fixed point refinement," in Proc. ACM/IEEE Design Automation Test Eur. Conf., 1999, pp. 271-276.

[4] K. Kum and W. Sung, "Combined word-length optimization and highlevel synthesis of digital signal processing systems," IEEE Trans. Comput.-Aided Des. Integr. Circuits Syst., vol. 20, no. 8, pp. 921-930, Aug. 2001.

[5] S. Kim and W. Sung, "Fixed-point error analysis and word length optimization of $8 \times 8$ IDCT architectures," IEEE Trans. Circuits Syst. Video Technol., vol. 8, no. 8, pp. 935-940, Dec. 1998.

[6] C. Shi and R. Brodersen, "Automated fixed-point data-type optimization tool for signal processing and communication systems," in Proc. ACM/IEEE Design Automation Conf., 2004, pp. 478-483.

[7] M. Willems, V. Bürgens, H. Keding, T. Grötker, and H. Meyr, "System level fixed-point design based on an interpolative approach," in Proc. ACM/IEEE Design Automation Conf., 1997, pp. 293-298.

[8] G. Constantinides, P. Cheung, and W. Luk, "Wordlength optimization for linear digital signal processing," IEEE Trans. Comput.-Aided Des. Integr. Circuits Syst., vol. 22, no. 10, pp. 1432-1442, Oct. 2003.

[9] C. Fang, R. Rutenbar, and T. Chen, "Fast, accurate static analysis for fixed-point finite-precision effects in DSP designs," in Proc. ACM/IEEE Int. Conf. Comput.-Aided Des., 2003, pp. 275-282.

[10] C. Fang, R. Rutenbar, M. Püschel, and T. Chen, "Toward efficient static analysis of finite-precision effects in DSP applications via affine arithmetic modeling," in Proc. ACM/IEEE Design Automation Conf., 2003, pp. 496-501.

[11] D. Menard and O. Sentieys, "Automatic evaluation of the accuracy of fixed-point algorithms," in Proc. ACM/IEEE Design Automation Test Eur. Conf., 2002, pp. 1530-1591.

[12] S. Wadekar and A. Parker, "Accuracy sensitive word-length selection for algorithm optimization," in Proc. IEEE Int. Conf. Comput. Des., 1998, pp. 54-61.

[13] L. de Figueiredo and J. Stolfi, "Self-validated numerical methods and applications," in Brazilian Mathematics Colloquium Monograph. Rio de Janeiro, Brazil: IMPA, 1997. Available: http://www.ic.unicamp.br/ stolfi/EXPORT/bibliography/FromBib.html\#fig-sto-97-iaaa

[14] V. Lefevre, J. Muller, and A. Tisserand, "Toward correctly rounded transcendentals," IEEE Trans. Comput., vol. 47, no. 11, pp. 1235-1243, Nov. 1998.

[15] M. J. Schulte and E. E. Swartzlander, Jr., "Hardware designs for exactly rounded elementary functions," IEEE Trans. Comput., vol. 43, no. 8, pp. 964-973, Aug. 1994.

[16] A. Nayak, M. Haldar, A. Choudhary, and P. Banerjee, "Precision and error analysis of Matlab applications during automated hardware synthesis for FPGAs," in Proc. DATE, 2001, pp. 722-728.

[17] M. Haldar, A. Nayak, N. Shenoy, A. Choudhary, and P. Banerjee, "FPGA hardware synthesis from Matlab," in VLSI Symp. Tech. Dig., 2001, pp. 299-304.

[18] G. Constantinides, "Perturbation analysis for word-length optimization," in Proc. IEEE Symp. Field-Program. Custom Comput. Machines, 2003, pp. 81-90.

[19] O. Mencer, D. Pearce, L. Howes, and W. Luk, "Design space exploration with a stream compiler," in Proc. IEEE Int. Conf. Field-Program. Technol., 2003, pp. 270-277.

[20] Xilinx Inc., Xilinx System Generator User Guide v6.3, (2004). [Online]. Available: http://www.xilinx.com

[21] L. Ingber, "Very fast simulated re-annealing," J. Math. Comput. Model., vol. 12, no. 8, pp. 967-973, 1989.

[22] —- Adaptive Simulated Annealing (ASA) 25.15, (2004). [Online]. Available: http://www.ingber.com/\#ASA

[23] R. Moore, Interval Analysis. Englewood Cliffs, NJ: Prentice-Hall, 1966.

[24] A. Skodras, C. Christopoulos, and T. Ebrahimi, "The JPEG 2000 still image compression standard," IEEE Signal Process. Mag., vol. 18, no. 5, pp. 36-58, Sep. 2001.

[25] V. Strassen, "Gaussian elimination is not optimal," Numer. Math., vol. 13, no. 4, pp. 354-356, 1969.

[26] J. Jiang, W. Luk, and D. Rueckert, "FPGA-based computation of freeform deformations in medical image registration," in Proc. IEEE Int. Conf. Field-Program. Technol., 2003, pp. 234-241.

[27] A. Madisetti and A. N. Willson, Jr., "A $100 \mathrm{MHz}$ 2-D $8 \times 8$ DCT/IDCT processor for HDTV applications," IEEE Trans. Circuits Syst. Video Technol., vol. 5, no. 2, pp. 158-165, Apr. 1995.

[28] ILOG SA, ILOG CPLEX 9.0, User's Manual, (2003). [Online]. Available: http://www.ilog.com/products/cplex

[29] G. Constantinides, P. Cheung, and W. Luk, "Optimum wordlength allocation," in Proc. IEEE Symp. Field Program. Custom Comput. Machines, 2002, pp. 219-228.

[30] R. Gonzalez, "Xtensa: A configurable and extensible processor," IEEE Micro., vol. 20, no. 2, pp. 60-70, Mar./Apr. 2000.

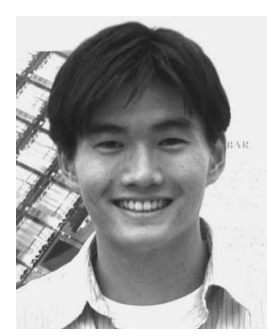

Dong-U. Lee ( $\left.\mathrm{S}^{\prime} 01-\mathrm{M}^{\prime} 05\right)$ received the B.Eng. degree in information systems engineering and the $\mathrm{Ph} . \mathrm{D}$. degree in computing, both from Imperial College London, U.K., in 2001 and 2004, respectively.

$\mathrm{He}$ is currently a Postdoctoral Researcher at the Electrical Engineering Department, University of California, Los Angeles, where he is working on channel codes and symbol timing synchronization for deep-space communications with Jet Propulsion Laboratory, NASA. His research interests include computer arithmetic, communications, design automation, reconfigurable computing, and video image processing.

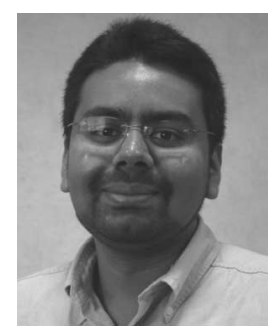

Altaf Abdul Gaffar (S'03-M'05) received the B.Eng. degree in information systems engineering and the Ph.D. degree in computing, both from Imperial College London, U.K., in 2000 and 2005, respectively.

He is currently working as a Research Associate at the Electrical and Electronic Engineering Department, Imperial College London. His research interests include bit-width optimization for floating-point and fixed-point arithmetic, and high-level power estimation and optimization techniques. 


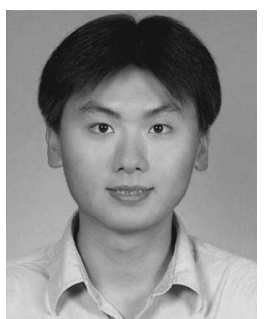

Ray C. C. Cheung (S'01) received the B.Eng. and M.Phil. degrees in computer engineering and computer science and engineering from the Chinese University of Hong Kong (CUHK), China, in 1999 and 2001, respectively. He is currently working toward the Ph.D. degree with the Custom Computing group, Department of Computing, Imperial College London, U.K.

In 2001, he worked as a System Administrator in the Center of Large-Scale Computation (CLC) of Cluster Technology, Hong Kong. From January 2002 to December 2003, he was an Instructor of the Department of Computer Science and Engineering, CUHK. His current research interests include computerarithmetic hardware designs and design exploration of system-on-chip (SoC) designs and embedded systems.

Mr. Cheung is a Student Member of the Association for Computing Machinery (ACM) and is the Newsletter and Web Editor of the Special Interest Group on Design Automation (SIGDA) U.K. chapter.

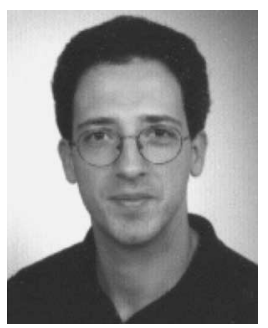

Oskar Mencer (M'96) received the B.S. degree in computer engineering from The Technion, Israel, in 1994 and the M.S. and Ph.D. degrees in electrical engineering from Stanford University, Stanford, CA, in 1997 and 2000, respectively.

He founded MAXELER Technologies, in 2003, after three years as a member of Technical Staff in the Computing Sciences Research Center at Bell Labs. He is a member of the academic staff in the Department of Computing, Imperial College London, U.K., and with the Custom Computing group. His research interests include computer architecture, computer arithmetic, very large scale integration (VLSI) microarchitecture, VLSI computer-aided design (CAD), and reconfigurable (custom) computing. More specifically, he is interested in exploring application-specific representation of computation at the algorithm level, the architecture level, and the arithmetic level.

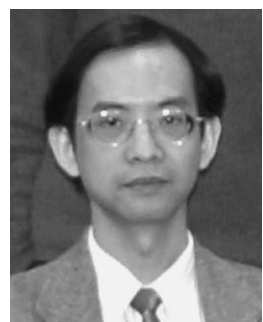

Wayne Luk (S'85-M'89) received the MA, M.Sc., and D.Phil. degrees in engineering and computing science from the University of Oxford, Oxford, U.K, in 1984,1985 , and 1989 , respectively.

$\mathrm{He}$ is a Professor of Computer Engineering in the Department of Computing, Imperial College London, U.K., and leads the Custom Computing Group there. His research interests include theory and practice of customizing hardware and software for specific application domains, such as graphics and image processing, multimedia, and communications. Much of his current work involves high-level compilation techniques and tools for parallel computers and embedded systems, particularly those containing reconfigurable devices such as field-programmable gate arrays.

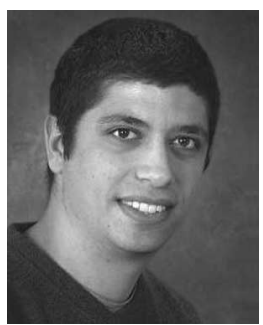

George A. Constantinides (S'96-M'01) received the M.Eng. degree in information systems engineering and the Ph.D. degree in electrical and electronic engineering from Imperial College London, U.K., in 1998 and 2001, respectively.

Since 2002, he has been a Lecturer in Digital Systems at the Electrical and Electronic Engineering Department, Imperial College London. He is the author of Synthesis and Optimization of DSP Algorithms (Dordrecht, Germany: Kluwer, 2004). His research interests include reconfigurable computing and electronic design automation, with a particular focus on digital signal processing algorithms.

Dr. Constantinides was program Cochair of the International Conference on Field-Programmable Logic and Applications, in 2003, and serves on the Program Committees of Field-Programmable Logic and Applications (FPL), Field-Programmable Technology (FPT), International Symposium on Circuits and Systems (ISCAS), Engineering of Reconfigurable Systems and Algorithms (ERSA), and Applied Reconfigurable Computing (ARC). He was the Founding Chair of the Special Interest Group on Design Automation (SIGDA) U.K. Chapter, serving as General Chair, in 2001, and Technical Chair of the annual workshop, from 2002 to 2003. He is a member of the Association for Computing Machinery. 